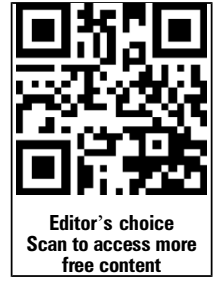

- Additional material is published online only. To view please visit the journal online (http://dx.doi.org/10.1136/ heartjnl-2015-307657).

For numbered affiliations see end of article.

\section{Correspondence to} Dr Annette Schophuus Jensen, Department of Cardiology, section 2014, Rigshospitalet, Blegdamsvej 9, Copenhagen 2100, Denmark; schophuus@gmail.com

Received 11 February 2015 Revised 23 April 2015 Accepted 4 May 2015

Published Online First 5 June 2015

\section{SLinked}

- http://dx.doi.org/10.1136/ heartjnl-2015-307849

http://dx.doi.org/10.1136/

heartjnl-2015-308045

\section{CrossMark}

To cite: Jensen AS, Idorn L, Thomsen C, et al. Heart 2015:101:1540-1546.

\title{
Prevalence of cerebral and pulmonary thrombosis in patients with cyanotic congenital heart disease
}

\author{
A S Jensen, ${ }^{1} \mathrm{~L}$ Idorn, ${ }^{1} \mathrm{C}$ Thomsen, ${ }^{2} \mathrm{P}$ von der Recke, ${ }^{3} \mathrm{~J}$ Mortensen, ${ }^{4} \mathrm{~K}$ E Sørensen, ${ }^{5}$ \\ U Thilén, ${ }^{6}$ E Nagy, ${ }^{7}$ K F Kofoed, ${ }_{1}^{1}$ S R Ostrowski, ${ }^{8}$ L Søndergaard ${ }^{1}$
}

\begin{abstract}
Background Patients with cyanotic congenital heart disease (CCHD) have a high prevalence of thrombosis, the most frequently described locations being the cerebral and pulmonary vessels. The reported prevalence of both cerebral infarction and pulmonary thrombosis has been highly variable. The aim of this study was to examine the prevalence of both cerebral and pulmonary thrombosis in CCHD according to medical history and imaging. In addition, the role of known erythrocytosis and haemostatic abnormalities as risk factors was evaluated.
\end{abstract}

Methods and results A cross-sectional descriptive study examining 98 stable adult patients with CCHD with a medical questionnaire, blood samples, MRI of the cerebrum ( $n=72$ ), multidetector CT imaging (MDCT) of the thorax $(n=76)$ and pulmonary scintigraphy (ventilation/perfusion/single-photon emission computerised tomography/CT) $(n=66)$. The prevalence of cerebral infarction and pulmonary thrombosis according to imaging were $47 \%$ and $31 \%$, respectively. Comparing the findings with previous medical history revealed a large under-reporting of thrombosis with only $22 \%$ of the patients having a clinical history of stroke and $25 \%$ of pulmonary thrombosis. There was no association between the degree of erythrocytosis or haemostatic abnormalities and the prevalence of thrombosis.

Conclusions Patients with CCHD have a prevalence of both cerebral and pulmonary thrombosis of around $30 \%-40 \%$, which is much higher than that reported previously. Furthermore, there is a large discrepancy between clinical history and imaging findings, suggesting a high prevalence of silent thrombotic events. Neither erythrocytosis nor haemostatic abnormalities were associated with the prevalence of thrombosis in patients with CCHD.

Trial registration number http://www.cvk.sum.dk/ CVK/Home/English.aspx (H-KF-2006-4068).

\section{INTRODUCTION}

Patients with cyanotic congenital heart disease (CCHD) have a high prevalence of thrombosis despite their relatively young age and the absence of classical cardiovascular risk factors. ${ }^{1}{ }^{2}$ Cerebral infarction and pulmonary thrombosis are reported as the most common manifestations, although the prevalence is uncertain. Cerebral infarction has been found to be $5 \%$ in one study, whereas other studies describe a prevalence as high as $23 \% .{ }^{1-4}$ Similar discrepancies exist in regard to pulmonary thrombosis. ${ }^{5-8}$
Besides the uncertain prevalence, the cause of cerebral and pulmonary thrombosis in CCHD is unknown. Prothrombotic conditions such as dilated and slow-flow cardiac chambers and vessels and arrhythmias may only be part of the explanation. ${ }^{79}$ Elevated haematocrit secondary to hypoxaemia and the presence of haemostatic abnormalities have also been suggested as contributors to thrombogenesis. ${ }^{10-12}$

Therefore, this study was conducted to determine the prevalence of cerebral infarction and pulmonary thrombosis in adult patients with CCHD according to both clinical history and imaging and to examine whether the increased prevalence could be associated with secondary erythrocytosis and/or haemostatic abnormalities.

\section{METHODS}

\section{Study design}

This was a cross-sectional descriptive multicentre study.

\section{Patients}

Between February 2007 and August 2010 clinically stable adult patients with CCHD followed up at the University Hospitals in Lund and Stockholm, Sweden, as well as Aarhus University Hospital Skejby and at Rigshospitalet, Denmark, were invited to participate in the study. CCHD was defined as the presence of a congenital heart defect causing bidirectional or right-to-left shunting with systemic oxygen saturation at rest of $<92 \%$ and/or $<87 \%$ during exercise. ${ }^{7}$ All patients enrolled in the study were referred to Rigshospitalet in Copenhagen, where all blood tests, medical history/medical questionnaire and imaging were performed.

\section{Blood samples}

Blood was drawn from an antecubital vein into Vacutainer tubes containing EDTA for the whole blood (WB) count, heparin for the iron status and citrate for the thrombelastography (TEG).

\section{Blood count and iron status}

Haemoglobin, haematocrit, platelets, mean corpuscular volume and mean corpuscular haemoglobin concentration were measured on the SYSMEX XE 2100 system. Iron, ferritin and transferrin saturation were analysed on the Modular P system.

\section{Thrombelastography}

TEG is a WB analysis, which reflects the different phases of the haemostatic process. TEG was 
performed in accordance with the manufacturer's instructions. All samples were analysed within $30 \mathrm{~min}$ after the blood sample was drawn using a TEG coagulation analyser (5000 series TEG analyser; Haemoscope Corporation, Chicago, Illinois, USA).

\section{Medical history}

Data regarding previous cerebral infarction or pulmonary thrombosis was obtained through a medical history questionnaire and confirmed by medical records. An event was defined as clinical symptoms of cerebral or pulmonary thrombosis causing hospitalisation and discharge with the diagnosis cerebral infarction or pulmonary thrombosis, but not necessarily verified by imaging modalities.

\section{MRI of the brain}

MRI of the brain was undertaken with a 3T Siemens Magnetom Trio Tim syngo B17 scanner. T1-weighted images were obtained using a FLAIR (fluid-attenuated inversion recovery) sequence (repetition time $(\mathrm{TR})=2 \mathrm{~s}$, echo time $(\mathrm{TE})=0.011 \mathrm{~s}$, inversion time $(\mathrm{TI})=0.8 \mathrm{~s})$ where images of the whole brain were acquired with $0.005 \mathrm{~m}$ sagittal slice thickness and interslice distance of $30 \%(0.0015 \mathrm{~m})$. Axial T2 turbo spin-echo images $(\mathrm{TR}=4 \mathrm{~s}$, $\mathrm{TE}=0.089 \mathrm{~s})$ slice thickness $0.005 \mathrm{~m}$, interslice distance $20 \%$ $(0.001 \mathrm{~m})$ and FLAIR $(\mathrm{TR}=9 \mathrm{~s}, \mathrm{TE}=0.090 \mathrm{~s}, \mathrm{TI}=2.5 \mathrm{~s})$ of the whole brain were acquired using $0.003 \mathrm{~m}$ slices and interslice distance $10 \%(0.0003 \mathrm{~m})$ respectively. Echo planar imaging diffusion-weighted images in the axial plane of the whole brain were acquired in $0.005 \mathrm{~m}$ slices and interslice distance $30 \%$ $(0.0015 \mathrm{~m})$ with a diffusion encoding in three directions of $\mathrm{b}=0,500.000 .000$ and 1.000.000.000 $\mathrm{s} / \mathrm{m}^{2}$. An ADC (apparent diffusion coefficient) image was calculated from the three diffusion-weighted images. Cortical infarctions were seen as areas with regional cortical thinning, widened sulci and subcortical white matter (WM) signal changes. Lacunar infarcts were seen in the deep WM as lacuna with signal as cerebrospinal fluid often with a rim of WM hyperintensity on FLAIR images. Acute infarcts were identified as lesions with decreased ADC values.

\section{Multidetector CT imaging of thorax}

Image acquisition was initially performed using a 64-slice multidetector CT imaging scanner (MDCT) (Toshiba Aquilion 64, Japan) until June 2009, when the scanner was replaced with a 320-slice MDCT/volume scanner. The protocol and scanning parameters for both scanners consisted of a non-gated helical angio, triggered in the pulmonary trunk for visualisation of arterial pulmonary vasculature. Image acquisition was performed according to the recommendations of the manufacturer $-64 \times 0.5 \mathrm{~mm}$ detector collimation, $120 \mathrm{kV}$ tube voltage, A-modulation tube current (thus A ranging between 0.15 and $0.3 \mathrm{~A}$, depending on body mass) with a gantry rotation time of $0.4 \mathrm{~s}$. Intravenous contrast medium was infused, using automated bolus tracking in the pulmonary truncus at the level of $160 \mathrm{HU}$ (Hounsfield Units) (Visipaque 320, GE Healthcare), with a flow rate of $0.004 \mathrm{~L} / \mathrm{s}$, total of $0.05-0.06 \mathrm{~L}$ (weight $<60 \mathrm{~kg}$ received only $0.05 \mathrm{~L}$ ), followed by a saline chaser of $0.04 \mathrm{~L} / \mathrm{s}$. Images were reconstructed with $0.0005 \mathrm{~m}$ slice thickness and increments of $0.0003 \mathrm{~m}$ or $0.0005 \mathrm{~m}$. The reconstruction was made with a dedicated soft tissue filter/algorithm and a lung filter. The MDCT images were used to identify thromboses, determine mural calcifications of the pulmonary arteries and measure the diameter of the pulmonary trunk and the main pulmonary arteries in order to determine dilatation/aneurysm. The criteria used for the evaluation of pulmonary dilatation (pulmonary trunk
$>0.024 \mathrm{~m}$ and/or right pulmonary artery $>0.016 \mathrm{~m}$ ) or pulmonary aneurysm (pulmonary trunk $>0.048 \mathrm{~m}$ and/or right pulmonary artery $>0.032 \mathrm{~m}$ ) were published norms in young healthy people, previously used by Perloff $e t a l^{6}$ in evaluating MDCT in patients with Eisenmenger syndrome. ${ }^{13}$

\section{Pulmonary scintigraphy}

Pulmonary ${ }^{81 \mathrm{M}} \mathrm{Kr}$ ventilation and ${ }^{99 \mathrm{M}} \mathrm{Tc}$-macroaggregated albumin perfusion (ventilation/perfusion, $\mathrm{V} / \mathrm{Q}$ ) scintigraphy was performed as planar imaging in six projections in the first year and in later years as single-photon emission computerised tomography (SPECT)/CT using an integrated two-headed gamma camera and an MDCT (16-slice) scanner (Philips Precedence, Philips Healthcare, Eindhoven, the Netherlands).

The SPECT included a perfusion and a ventilation part obtained simultaneously within $780 \mathrm{~s}$ (ie, 36 projections per head of $12-20 \mathrm{~s}$ each over $180^{\circ}, 128 \times 128$ matrix, low-energy, general-purpose collimators). It was performed immediately after a low-dose CT scan $(\mathrm{kVp}$ 140, mAs/slice 20, collimator $0.016 \times 0.0015 \mathrm{~m}$, rotation time $0.5 \mathrm{~s}$, pitch 0.813 ), which as the SPECT was obtained during tidal breathing. SPECT data sets were attenuation corrected using the low-dose CT acquisition with iterative reconstruction using the software Autospect+ and Astonish with three iterations and 16 subsets (Philips Medical Systems, Eindhoven, the Netherlands). V/Q-planar and SPECT scans were read on an Extended Brilliance Workspace-Nuclear Medicine (EBW-NM) workstation (Philips Medical Systems, Eindhoven, the Netherlands). All perfusion and ventilation defects were noted for size and segmental location. Pulmonary embolism (PE) was diagnosed, according to the European Association for Nuclear Medicine guidelines for V/Q (SPECT) and MDCT to detect PE, if one or more wedge-shaped segmental sized (or larger) perfusion defects with normal ventilation (mismatch) were present. ${ }^{14}$ If the low-dose CT showed parenchymal defects in an area with subtle perfusion defects PE was excluded. An automatic 3D imaging registration software tool was used to display the low-dose CT, V/Q SPECT, and fusion images in axial, coronal and sagittal planes (Fusion viewer, EBW-NM, Philips Medical Systems).

All imaging results (MRI, MDCT and V/Q SPECT/CT) were read blinded to previous medical history of the patients except previous cardiac surgery/diagnosis.

\section{Statistical analysis}

Statistical analysis was conducted with Predictive Analytics Software statistics V.18.0. Numerical values are expressed as mean and SD unless otherwise stated. Continuous variables were compared using a Student's $t$ test when normally distributed or Mann-Whitney U test otherwise. Categorical variables were compared by $\chi^{2}$ or Fisher's exact test. To assess the association between variables, predefined variables reported in previous studies (age, gender, saturation, iron deficiency (defined as ferritin $<12 \mu \mathrm{g} / \mathrm{L}$ and/or transferrin saturation $<20 \%$ ), arrhythmia, previous heart surgery, pulmonary arterial hypertension $(\mathrm{PAH})$, anticoagulation and complexity of CCHD) were examined with simple logistic regression. If variables were statistically significant, multiple regression was performed. A p value $<0.05$ was considered statistically significant.

\section{Ethics}

The study was conducted according to the Declaration of Helsinki. The Danish Ethical Committee approved the protocol (H-KF-2006-4068). Written informed consent was obtained from all patients. 


\section{RESULTS}

One hundred and two patients with CCHD were invited to participate in the study. Ninety-eight patients accepted and were examined with blood samples and a medical questionnaire. Due to lack of ability to cooperate-for example, Down syndrome, contrast allergy, pacemaker, claustrophobia and fear of radiation - the number of patients who underwent a cerebral MRI were 72, thoracic MDCT 76 and V/Q scintigraphy 66. Fifty-seven patients were examined with all three imaging modalities, pulmonary imaging (MDCT and V/Q SPECT/CT) was performed in 64 patients and 68 patients had both brain and lungs (MDCT or $\mathrm{V} / \mathrm{Q}$ SPECT/CT) examined. Demographic data are summarised in table 1.

Table 1 Demographics of the patients with cyanotic congenital heart disease

\begin{tabular}{|c|c|}
\hline Clinical characteristics & $\mathrm{N}=98$ \\
\hline Female gender, $\mathrm{N}(\%)$ & $55(56)$ \\
\hline Age, years & $40 \pm 14$ \\
\hline Saturation, \% & $83 \pm 7$ \\
\hline \multicolumn{2}{|l|}{ Diagnoses } \\
\hline Eisenmenger syndrome, $\mathrm{N}(\%)$ & $69(70)$ \\
\hline Ventricular septal defect, $\mathrm{N}$ & 47 \\
\hline Atrial septal defect, $\mathrm{N}$ & 12 \\
\hline Atrioventricular septal defect, $\mathrm{N}$ & 5 \\
\hline Persistent ductus arteriosus, $\mathrm{N}$ & 4 \\
\hline Aortopulmonary window, $\mathrm{N}$ & 1 \\
\hline Pulmonary arteriovenous malformation, $\mathrm{N}(\%)$ & $2(2)$ \\
\hline Univentricular heart, $\mathrm{N}(\%)$ & $17(18)$ \\
\hline $\begin{array}{l}\text { Pulmonary atresia, ventricular septal defect and } \\
\text { major aortopulmonary collateral arteries, } \mathrm{N}(\%)\end{array}$ & $9(9)$ \\
\hline Ebstein anomaly with atrial septal defect, N (\%) & $1(1)$ \\
\hline Down syndrome, N (\%) & $14(14)$ \\
\hline 22q11.2 deletion syndrome (DiGeorge syndrome), N (\%) & $6(6)$ \\
\hline Pulmonary hypertension*, N (\%) & $83(85)$ \\
\hline \multicolumn{2}{|l|}{ Medical history } \\
\hline Previous history of thrombosis, N (\%) & $20(20) \dagger$ \\
\hline Brain, $\mathrm{N}$ & 13 \\
\hline Heart, N & 1 \\
\hline Lung, $\mathrm{N}$ & 6 \\
\hline Arm/leg, $\mathrm{N}$ & 1 \\
\hline Atrial flutter/fibrillation, N (\%) & $24(24)$ \\
\hline Paroxysmal, N (\%) & $18(18)$ \\
\hline Permanent, n (\%) & $6(6)$ \\
\hline Pacemaker, n (\%) & $7(7)$ \\
\hline Iron deficiency, n (\%) & $32(33)$ \\
\hline Previous phlebotomy, n (\%) & $32(33)$ \\
\hline Previous cardiac surgery, N (\%) & $19(20)$ \\
\hline \multicolumn{2}{|l|}{ Medication } \\
\hline Iron treatment, n (\%) & $12(12)$ \\
\hline Antiplatelet/coagulation medicine, n (\%) & $33(34) \ddagger$ \\
\hline Aspirin, $n(\%)$ & $15(16)$ \\
\hline Clopidogrel, n (\%) & $1(1)$ \\
\hline Warfarin, $\mathrm{n}(\%)$ & $15(16)$ \\
\hline Marcoumar, n (\%) & $6(6)$ \\
\hline
\end{tabular}

The results are shown as mean \pm SD or actual number and percentage. *Pulmonary hypertension had previously been verified by right heart catheterisation. tSome of the patients had had more than one thrombosis.

$\ddagger$ Some of the patients received more than one antiplatelet/coagulation medicine.

\section{Medical history}

According to medical history, 20\% $(n=20 / 98)$ of the patients had a medical history of one or more thromboses (table 1 ).

MRI of the brain

The imaging revealed that $47 \%(n=34 / 72)$ of the examined patients had radiological findings consistent with a previous stroke. In $53 \%(n=18 / 34)$ of the patients, more than one cerebral infarction was present. The cerebral infarctions were located in both the lacunar and cortical areas, and almost one-third of the patients had both types of infarctions. No acute infarctions were found, but a very high prevalence of subependymally located infarctions was seen (table 2, figure 1).

Pathological prevalence of WM hyperintensity lesions (WMHL) was seen in $65 \%(n=47 / 72)$ of the examined patients, irrespective of history/imaging verified infarction (table 2, online supplementary figure S1a).

Comparing the MRI results with medical history, only 21\% $(n=7 / 34)$ of the patients with a cerebral infarction on the MRI had a clinical history of stroke, whereas only $58 \%(n=7 / 12)$ of the patients with a previous history of stroke had a verified infarction on the MRI (table 3).

\section{MDCT of thorax}

Imaging illustrated that $20 \% \quad(n=15 / 76)$ of the examined patients with CCHD had pulmonary thrombosis. The thromboses were located both proximally and peripherally in the pulmonary arteries, and a combination was present in half of the patients. The thromboses were both mural and occluding. Other findings were that $97 \%(n=74 / 76)$ of the patients had dilated proximal pulmonary arteries including $21 \%(n=16 / 76)$ with aneurysmal dilatation. Mural calcifications of the pulmonary arteries were present in 34\% $(n=26 / 76)$. The majority of these patients also had $\mathrm{PAH}$ (table 4 , figure 2).

\section{V/Q SPECT/CT of the lungs}

The prevalence of pulmonary thrombosis was 29\% $(n=19 / 66)$ according to V/Q SPECT/CT. The distribution of pulmonary thromboses was equal between the apical and medial parts, and slightly less in the basal part of the right lung, whereas in the left lung the majority of thromboses were located in the apical

Table 2 The demographics and prevalence of cerebral thrombosis in patients with cyanotic congenital heart disease

\begin{tabular}{ll}
\hline Clinical characteristics & $\mathbf{n}=\mathbf{7 2}$ \\
\hline Imaging & \\
White matter hyperintensity lesions, $\mathrm{n}(\%)$ & $47(65)$ \\
Cerebral infarction, $\mathrm{n}(\%)$ & $34(47)$ \\
$\begin{array}{l}\text { Number of infarctions } \\
\mathrm{n}=1\end{array}$ & 16 \\
$\mathrm{n}=2$ & 6 \\
$\mathrm{n}=3$ & 5 \\
$\mathrm{n}>3$ & 7 \\
Type of infarction & \\
Lacunar, $\mathrm{n}(\%)$ & $14(42)$ \\
$\quad$ Subependymal located infarctions, $\mathrm{n}$ & 10 \\
Lacunar and cortical infarctions, $\mathrm{n}(\%)$ & $10(29)$ \\
Cortical, $\mathrm{n}$ (\%) & $10(29)$ \\
$\quad$ Posterior inferior cerebellar artery, $\mathrm{n}$ & 16 \\
\hline The results are shown as actual number and percentage.
\end{tabular}




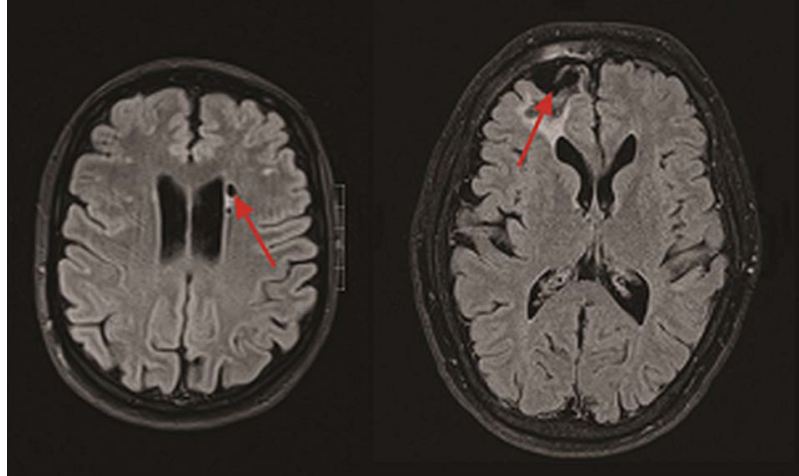

Figure 1 Magnetic resonance images (FLAIR sequence) of the brain a subependymally infarction (arrow left), and a frontal cortical infarction (arrow right).

part of the lung. The perfusion distribution between the apical, medial and basal parts of the two lungs was almost equal. A mottled pattern representing a possible patchy loss of perfusion was present in 67\% $(n=44 / 66)$ of the patients (table 4, online supplementary figure $\mathrm{S} 2 \mathrm{a}$ ).

The overall prevalence of pulmonary thrombosis, occluding and/or mural, detected by either MDCT or V/Q SPECT/CT, was $31 \%(n=24 / 78)$. In patients who had both imaging modalities performed the prevalence was slightly higher, at $33 \%$ $(n=21 / 64)$. In $48 \%(n=10 / 21)$ of these patients thrombosis was identified with both imaging techniques, where 33\% $(n=7 / 21)$ was diagnosed with V/Q SPECT/CT and 19\% $(n=4 / 21)$ with MDCT only.

Unlike patients with cerebral infarction, all patients with a medical history of pulmonary thrombosis had imaging confirming the diagnosis. However, these patients represented only $25 \%(n=6 / 24)$ of the total number of patients with radiological signs of pulmonary thrombosis (table 5).

Finally, comparing the findings from patients who had both brain and pulmonary imaging, only about a quarter of the

Table 3 The prevalence of cerebral thrombosis according to medical history and imaging

\begin{tabular}{lrrr}
\hline & \multicolumn{2}{l}{$\begin{array}{l}\text { Infarct on } \\
\text { cerebral MRI }\end{array}$} & \\
\cline { 2 - 3 } & No & Yes & Total \\
\hline Medical history & & & \\
No & & & \\
n & 33 & 27 & 60 \\
Percentage within medical history & 55 & 45 & 100 \\
Percentage within total infarct on MRI & 87 & 79 & 83 \\
Percentage of total & 46 & 37 & 83 \\
Yes & & & \\
n & 5 & 7 & 12 \\
Percentage within medical history & 42 & 58 & 100 \\
Percentage within total infarct on MRI & 13 & 21 & 17 \\
Percentage of total & 7 & 10 & 17 \\
Total & & & \\
n & 38 & 34 & 72 \\
Percentage within medical history & 53 & 47 & 100 \\
Percentage within total infarct on MRI & 100 & 100 & 100 \\
Percentage of total & 53 & 47 & 100 \\
\hline
\end{tabular}

Table 4 The demographics and prevalence of pulmonary thrombosis examined with $\mathrm{CT}$ (MDCT) and pulmonary scintigraphy (V/Q SPECT/CT) in patients with CCHD

\begin{tabular}{ll}
\hline Clinical characteristics & \\
\hline CT (MDCT), $n$ & 76 \\
Pulmonary thrombosis, $\mathrm{n}(\%)$ & $15(20)$ \\
Proximal thrombosis, $\mathrm{n}(\%)$ & $8(53)$ \\
Pulmonary trunk, $\mathrm{n}$ & 2 \\
Right pulmonary artery, $\mathrm{n}$ & 6 \\
Left pulmonary artery, $\mathrm{n}$ & 6 \\
Peripheral thrombosis, $\mathrm{n}(\%)$ & $11(73)$ \\
Right lung, $\mathrm{n}$ & 10 \\
Left lung, $\mathrm{n}$ & 6 \\
Mural thrombosis, $\mathrm{n}(\%)$ & $8(53)$ \\
Occluding thrombosis, $\mathrm{n}(\%)$ & $11(73)$ \\
Enlarged pulmonary arteries, $\mathrm{n}(\%)$ & $74(97)$ \\
Aneurysmal arteries, $\mathrm{n}(\%)$ & $16(21)$ \\
Calcification in the pulmonary vessels & $26(34)$ \\
Pulmonary trunk, $\mathrm{n}$ & 13 \\
Right pulmonary artery, $\mathrm{n}$ & 21 \\
Left pulmonary artery, $\mathrm{n}$ & 13 \\
Prevalence of pulmonary hypertension, $\mathrm{n}(\%)$ & $25(96)$ \\
Pulmonary scintigraphy (V/Q SPECT/CT), $\mathrm{n}$ & 66 \\
Pulmonary thrombosis, $\mathrm{n}(\%)$ & $19(29)$ \\
Apex of the right lung, $\mathrm{n}$ & 13 \\
Medial part of the right lung, $\mathrm{n}$ & 13 \\
Basal part of the right lung, $\mathrm{n}$ & 10 \\
Apex of the left lung, $\mathrm{n}$ & 12 \\
Medial part of the left lung, $\mathrm{n}$ & 8 \\
Basal part of the left lung, $\mathrm{n}$ & 6 \\
Mottled pattern on the perfusion scan, $\mathrm{n}(\%)$ & $44(67)$ \\
\hline &
\end{tabular}

The results are shown as actual number and percentage.

CCHD, cyanotic congenital heart disease; MDCT, multidetector CT imaging; V/Q/

SPECT, ventilation/perfusion/single-photon emission computerised tomography.

examined patients $(n=18 / 68)$ had both cerebral and pulmonary thromboses.

\section{Secondary erythrocytosis/haematological abnormalities}

Comparing the presence of cerebral infarction and/or pulmonary thrombosis detected by each imaging modality with the present haematocrit, platelet count, iron status and TEG, no significant difference was present between patients with and without thrombosis.

\section{Possible risk factors}

Complex CCHD and low oxygen saturation were both risk factors for cerebral infarction, individually (table 6) and in combination (OR 0.20 (0.05 to 0.75), p=0.02 and (HR 0.90 (0.83 to 0.99$), \mathrm{p}=0.02$ ). Patients with pulmonary thrombosis were associated with older age according to the MDCT findings, but not significant for V/Q SPECT/CT. No other variables were associated with increased risk of pulmonary or cerebral thrombosis (table 6).

\section{DISCUSSION}

This study demonstrated that despite knowledge of an increased prevalence of cerebral and pulmonary thrombosis in CCHD, this has been clinically underestimated. The reported prevalence of cerebral infarctions in this study is twice as high compared with previous studies. ${ }^{1-4}$ An explanation could be that in this 
Figure 2 MDCT of thorax of two patients with central located pulmonary thrombosis (green arrows right and red arrow left).

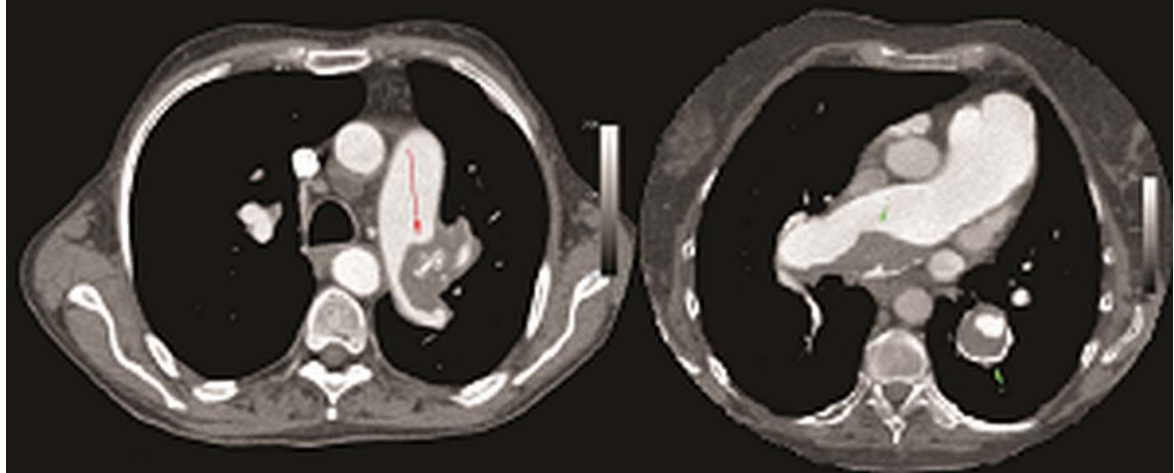

study CCHD patients in general were included and examined, and not only subgroups. Hoffmann et al has previously illustrated that patients with complex CCHD, for example, univentricular heart, have an increased prevalence of cerebral infarctions compared with patients with Eisenmenger syndrome. Similar results were found in this study, but as the majority of the examined patients were those with Eisenmenger syndrome (70\%), this alone could not explain the high prevalence. ${ }^{2}$ One explanation could have been that the patients were slightly older than in previous studies. However, in the present study, there was no correlation between age and prevalence of cerebral infarction. A more likely explanation may be that all patients in this study were examined with MRI, which may be more sensitive than clinical history to detect cerebral lesions. The large discrepancy between clinical history and actual findings on MRI in this study supports this assumption as well as the study of Horigome et al, which showed that clinical history and brain imaging are not very well correlated in patients with CCHD. ${ }^{15}$

The prevalence of pulmonary thrombosis in our study was, when reported according to the MDCT findings only,

Table 5 The prevalence of pulmonary thrombosis according to medical history and imaging

\begin{tabular}{lrrr}
\hline & \multicolumn{2}{c}{$\begin{array}{c}\text { Pulmonary } \\
\text { thrombosis } \\
\text { on imaging }\end{array}$} & \\
\cline { 2 - 3 } & No & Yes & Total \\
\hline Medical history & & & \\
No & & & \\
n & 54 & 18 & 72 \\
Percentage within medical history & 75 & 25 & 100 \\
Percentage within total pulmonary thrombosis & 100 & 75 & 92 \\
on imaging & & & \\
$\quad$ Percentage of total & 69 & 23 & 92 \\
Yes & & & \\
n & 0 & 6 & 6 \\
Percentage within medical history & 0 & 100 & 100 \\
$\quad$ Percentage within total pulmonary thrombosis & 0 & 25 & 8 \\
on imaging & & & \\
$\quad$ Percentage of total & 0 & 8 & 8 \\
Total & & & \\
n & 54 & 24 & 78 \\
Percentage within medical history & 69 & 31 & 100 \\
$\quad$ Percentage within total pulmonary thrombosis & 100 & 100 & 100 \\
on imaging & & & \\
Percentage total & 69 & 31 & 100 \\
\hline
\end{tabular}

comparable with previously reported findings by Broberg et $\mathrm{al}^{7}$ and Silversides et al. ${ }^{8}$ However, using V/Q alone or in combination with SPECT/CT showed a prevalence of PE almost 50\% higher than that reported with MDCT. The explanation is most likely that patients with CCHD in previous studies have been examined with MDCT. ${ }^{4-8}$ Gutte et al ${ }^{16}$ illustrated, when comparing the two methods, MDCT and V/Q SPECT/CT, that MDCT has a much lower sensitivity in diagnosing PE compared with V/Q SPECT/CT. Therefore, the higher prevalence in this study is most likely due to the use of a combination of the two imaging modalities, which were able to visualise both proximal non-occluding thromboses, which are normally not well visualised with V/Q SPECT/CT, as well as peripheral occluding thrombosis, which if located very distally, is not possible to be visualised with MDCT. This also explains why not all pulmonary thromboses were visualised with both imaging modalities.

Regarding pathogenesis of cerebral infarctions and pulmonary thrombosis in CCHD, this study confirmed the previously contested finding that secondary erythrocytosis and/or haemostatic abnormalities were not associated with increased prevalence of thrombotic events. ${ }^{1} 3491718$ Looking at possible risk factors, none of them were associated with both cerebral and pulmonary thrombosis, indicating possibly different aetiologies for the two types of thrombosis.

The location of cerebral infarction could lead to speculations regarding the pathogenesis. There was a very high prevalence of subependymal infarctions, which are uncommon, as well as a very high prevalence of WMHL. The subependymal area is located deep in the lacunar part of the brain and is supplied by long and very thin thaleostiate vessels. WMHL are well-known findings on MRI in most middle-aged and elderly people. When a large number of WMHL are present, or if the size of the lesions is large, these findings are considered pathological and interpreted as ischaemic lesions of the brain. ${ }^{19}$ Therefore, these findings could imply ischaemia and not thromboembolism as a possible explanation. Supporting this is the reported association between a cerebrovascular event and hypoxaemia, which has also been previously reported by Phornphutkul et al. ${ }^{20}$ Despite speculation, no conclusion can be made from this study due to the fact that there was also a trend between iron deficiency and infarction. Iron deficiency is a common finding in CCHD, it influences the haemostatic profile and microcytosis is a known independent risk factor of stroke in both children and adults. $^{1} 91820$

The reported clinical prevalence and the actual findings of both cerebral infarction and pulmonary thrombosis on imaging in this study revealed a significant under-reporting, indicating a high prevalence of 'silent thrombosis'. Patients with CCHD are used to being physically limited in daily life due to dyspnoea, 
Table 6 Simple logistic regression of previously described risk factors of cerebral and pulmonary thrombosis

\begin{tabular}{|c|c|c|c|c|c|c|}
\hline & \multicolumn{2}{|l|}{ MRI of brain } & \multicolumn{2}{|l|}{ MDCT thorax } & \multicolumn{2}{|l|}{ V/Q/SPECT/CT } \\
\hline & OR $(95 \% \mathrm{CI})$ & p Value & OR $(95 \% \mathrm{CI})$ & p Value & OR $(95 \% \mathrm{CI})$ & p Value \\
\hline Age & $1.03(0.99$ to 1.07$)$ & 0.14 & $1.05(1.00$ to 1.09$)$ & 0.04 & $1.01(0.97$ to 1.05$)$ & 0.74 \\
\hline Gender & $0.94(0.36$ to 2.46$)$ & 0.90 & $2.03(0.65$ to 6.34$)$ & 0.23 & $0.41(0.14$ to 1.22$)$ & 0.11 \\
\hline Saturation & $0.92(0.84$ to 1.00$)$ & 0.04 & $1.02(0.93$ to 1.12$)$ & 0.71 & $0.97(0.89$ to 1.07$)$ & 0.60 \\
\hline Iron deficiency & $0.39(0.14$ to 1.08$)$ & 0.07 & $1.44(0.41$ to 5.09$)$ & 0.57 & $2.33(0.67$ to 8.12$)$ & 0.19 \\
\hline Arrhythmia & $0.41(0.14$ to 1.22$)$ & 0.11 & $0.65(0.19$ to 2.21$)$ & 0.49 & $0.33(0.10$ to 1.04$)$ & 0.06 \\
\hline Previous heart surgery & $1.55(0.45$ to 5.28$)$ & 0.49 & $0.42(0.11$ to 1.63$)$ & 0.21 & $0.45(0.11$ to 1.89$)$ & 0.27 \\
\hline Pulmonary hypertension & $0.73(0.11$ to 4.65$)$ & 0.74 & $0.66(0.07$ to 5.89$)$ & 0.71 & $8.63(0.84$ to 89.0$)$ & 0.07 \\
\hline Anticoagulation & $0.43(0.15$ to 1.22$)$ & 0.11 & $1.05(0.32$ to 3.47$)$ & 0.94 & $0.71(0.24$ to 2.12$)$ & 0.54 \\
\hline Complex CCHD & $0.25(0.07$ to 0.87$)$ & 0.03 & $0.37(0.11$ to 1.23$)$ & 0.11 & $0.30(0.09$ to 1.03$)$ & 0.06 \\
\hline
\end{tabular}

and often have symptoms similar to a cerebral event, when hyperviscosity/iron deficiency is present. Therefore, perhaps, minor thrombotic events causing only a few symptoms are not registered as deterioration by patients. It is interesting that despite large under-reporting, all patients with a history of pulmonary thrombosis had verified pulmonary thrombosis on imaging, which was not the case with cerebral infarction. This could indicate that symptoms such as desaturation and increased dyspnoea perhaps are associated with a higher diagnostic use of blood/imaging modalities compared with cerebral symptoms such as dizziness, light-headedness and visual disturbance.

Finally, the high prevalence of thrombosis found in this study contributes to a discussion regarding antithrombotic/anticoagulation therapy in patients with CCHD. Anticoagulation is, according to guidelines, not recommended as routine treatment in CCHD. ${ }^{21}{ }^{22}$ It is, however, concerning to have such a high prevalence of thrombosis in a relatively young cohort of patients, and prevention may be warranted. This study indicates that the pathogenesis of cerebral infarction and that of pulmonary thrombosis may be different. Furthermore, despite the limitations mentioned below, no difference in prevalence of thrombosis could be shown between patients receiving anticoagulation and those who do not in this and a previous study. ${ }^{23}$ Therefore, antithrombotic/anticoagulation therapy in patients with CCHD remains open to speculation and warrants further investigation.

\section{Limitations}

A limitation of this study is that imaging is not able to determine when a patient has had the thrombotic event. Therefore, phlebotomy/microcytosis may be the cause of the high number of cerebral infarctions, but previous admissions with intravenous access, catheterisation and arrhythmias may also be possible explanations.

A similar conundrum is present when discussing antithrombotic/anticoagulation therapy. Many patients did not have an MRI or CT of the brain/thorax performed before initiating therapy, since indication for therapy was rarely a thrombotic event.

\section{CONCLUSION}

Patients with CCHD have a prevalence of both cerebral and pulmonary thrombosis of around $30 \%-40 \%$, which is much higher than previously reported. Furthermore, there is a large discrepancy between clinical history and imaging findings, suggesting a high prevalence of 'silent thrombotic events'. Finally, neither secondary erythrocytosis nor haemostatic abnormalities, which are common findings in CCHD, could explain the high prevalence of thrombosis in CCHD.

\section{Key messages}

What is already known on this subject?

Patients with cyanotic congenital heart disease (CCHD) have a high prevalence of thrombosis despite their relatively young age and the absence of classical cardiovascular risk factors.

What might this study add?

This study indicates, through systematic examination with imaging, regardless of previous medical history, that patients with CCHD have a prevalence of both cerebral and pulmonary thrombosis of around $30 \%-40 \%$.

How might this impact on clinical practice? Despite knowledge of a high prevalence of thrombotic events in CCHD, this study revealed that there is a large under-reporting/ misdiagnosis especially regarding cerebral events. Hopefully, this knowledge will contribute to a higher use of diagnostic imaging, if patients have possible neurological or pulmonary symptoms, in order to confirm/exclude the diagnosis.

\section{Author affiliations}

${ }^{1}$ Department of Cardiology, Copenhagen University Hospital, Rigshospitalet, Copenhagen, Denmark

${ }^{2}$ Department of Radiology, Copenhagen University Hospital, Rigshospitalet, Copenhagen, Denmark

${ }^{3}$ Amager Radiological Clinic, Copenhagen, Denmark

${ }^{4}$ Department of Clinical Physiology \& Nuclear Medicine, Copenhagen University Hospital, Rigshospitalet, Copenhagen, Denmark

${ }^{5}$ Department of Cardiology, Aarhus University Hospital, Skejby, Aarhus, Denmark ${ }^{6}$ Department of Cardiology, Lund University Hospital, Lund, Sweden

${ }^{7}$ Department of Cardiology, Karolinska University Hospital, Stockholm, Sweden

${ }^{8}$ Section for Transfusion Medicine, Capital Region Blood Bank, Copenhagen

University Hospital, Rigshospitalet, Copenhagen, Denmark

Contributors ASJ: Contributed to the design, recruitment of patients, examination, evaluation of results and writing of the paper. LI: Contributed to evaluation of results and critical review of the paper. CT: Contributed with MRI, evaluation of results and critical review of the paper. PvdR: Contributed with MDCT, evaluation of results and critical review of the paper. JM: Contributed with V/Q SPECT/CT, evaluation of results and critical review of the paper. KES: Contributed to the recruitment of patients, evaluation of results and critical review of the paper. UT: Contributed to the recruitment of patients, evaluation of results and critical review of the paper. EN: Contributed to the recruitment of patients, evaluation of results and 
critical review of the paper. KFK: Contributed to evaluation of results and critical review of the paper. SRO: Contributed with evaluations of TEG results and critical review of the paper. LS: Contributed to the design, funding of the study, recruitment of patients, evaluation of results and critical review of the paper.

Funding This work was supported by the Research Foundation of the Heart Centre, Rigshospitalet; The Aase og Ejnar Danielsen Foundation; The Foundation of King Christian the Tenth; The Research Foundation of the Danish Medical Association; The Beckett Foundation; Pfizer ApS and Actelion.

Competing interests None declared.

Ethics approval The Danish Ethical Committee (H-KF-2006-4068)http://www.cvk. sum.dk/CVK/Home/English.aspx(H-KF-2006-4068.

Provenance and peer review Not commissioned; externally peer reviewed.

\section{REFERENCES}

1 Ammash N, Warnes CA. Cerebrovascular events in adult patients with cyanotic congenital heart disease. J Am Coll Cardiol 1996;28:768-72.

2 Hoffmann A, Chockalingam P, Balint $\mathrm{OH}$, et al. Cerebrovascular accidents in adult patients with congenital heart disease. Heart 2010;96:1223-6.

3 Perloff JK, Marelli AJ, Miner PD. Risk of stroke in adults with cyanotic congenital heart disease. Circulation 1993;87:1954-9.

4 Daliento L, Somerville J, Presbitero P, et al. Eisenmenger syndrome. Factors relating to deterioration and death. Eur Heart J 1998;19:1845-55.

5 Caramuru LH, Maeda NY, Bydlowski SP, et al. Age-dependent likelihood of in situ thrombosis in secondary pulmonary hypertension. Clin Appl Thromb Hemost 2004; 10:217-23.

6 Perloff JK, Hart EM, Greaves SM, et al. Proximal pulmonary arterial and intrapulmonary radiologic features of Eisenmenger syndrome and primary pulmonary hypertension. Am J Cardiol 2003;92:182-7.

7 Broberg CS, Ujita M, Prasad S, et al. Pulmonary arterial thrombosis in Eisenmenger syndrome is associated with biventricular dysfunction and decreased pulmonary flow velocity. J Am Coll Cardiol 2007;50:634-42.

8 Silversides CK, Granton JT, Konen E, et al. Pulmonary thrombosis in adults with Eisenmenger syndrome. J Am Coll Cardiol 2003;42:1982-7.

9 Gatzoulis MA, Webb GD, Daubeney PEF. Eisenmenger's syndrome. Diagnosis and management of adult congenital heart disease. Edinburgh: Churchill Livingstone, Elsevier Limited, 2003:363-377.
10 Kajimoto H, Nakazawa M, Murasaki K, et al. Increased thrombogenesity in patients with cyanotic congenital heart disease. Circ J 2007;71:948-53.

11 Spivak JL. Polycythemia vera: myths, mechanisms, and management. Blood 2002;100:4272-90.

12 Horigome $H$, Murakami T, Isobe T, et al. Soluble P-selectin and thrombomodulin-protein C-Protein $S$ pathway in cyanotic congenital heart disease with secondary erythrocytosis. Thromb Res 2003;112:223-7.

13 Drexler M, Erbel R, Müller U, et al. Measurement of intracardiac dimensions and structures in normal young adult subjects by transesophageal echocardiography. Am J Cardiol 1990;65:1491-6.

14 Bajc M, Neilly JB, Miniati M, et al. EANM guidelines for ventilation/perfusion scintigraphy: Part 1. Pulmonary imaging with ventilation/perfusion single photon emission tomography. Eur J Nucl Med Mol Imaging 2009;36:1356-70.

15 Horigome $\mathrm{H}$, Iwasaki N, Anno I, et al. Magnetic resonance imaging of the brain and haematological profile in adult cyanotic congenital heart disease without stroke. Heart 2006;92:263-5.

16 Gutte H, Mortensen J, Jensen CV, et al. Detection of pulmonary embolism with combined ventilation-perfusion SPECT and low-dose CT: head-to-head comparison with multidetector CT angiography. J Nucl Med 2009;50:1987-92.

17 Shibata J, Hasegawa J, Siemens H-J, et al. Hemostasis and coagulation at a hematocrit level of 0.85: functional consequences of erythrocytosis. Blood 2003; 101:4416-22.

18 Jensen AS, Johansson PI, Idorn L, et al. The haematocrit-an important factor causing impaired haemostasis in patients with cyanotic congenital heart disease. Int J Cardiol 2013;167:1317-21.

19 Christiansen P, Larsson HB, Thomsen C, et al. Age dependent white matter lesions and brain volume changes in healthy volunteers. Acta Radiol 1994;35:117-22.

20 Phornphutkul C, Rosenthal A, Nadas AS, et al. Cerebrovascular accidents in infants and children with cyanotic congenital heart disease. Am J Cardiol 1973;32:329-34.

21 Warnes CA, Williams RG, Bashore TM, et al. ACC/AHA 2008 Guidelines for the Management of Adults with Congenital Heart Disease: a report of the American College of Cardiology/American Heart Association Task Force on Practice Guidelines. Circulation 2008;118:e714-833.

22 Baumgartner H, Bonhoeffer P, De Groot NMS, et al. ESC Guidelines for the management of grown-up congenital heart disease (new version 2010). Eur Heart J 2010;31:2915-57.

23 Sandoval J, Santos LE, Córdova J, et al. Does anticoagulation in Eisenmenger syndrome impact long-term survival? Congenit Heart Dis 2012;7:268-76. 